\title{
A checklist of marine larval trematodes (Digenea) in molluscs from Argentina, Southwestern Atlantic coast
}

\author{
Estefanía Bagnato, Carmen Gilardoni, Gisele Di Giorgio and Florencia Cremonte* \\ Laboratorio de Parasitología, Centro Nacional Patagónico (CONICET), Bvd. Brown 2915, U9120ACF Puerto Madryn, Argentina \\ * Corresponding author. E-mail: fcremont@cenpat-conicet.gob.ar
}

\begin{abstract}
A checklist of cercariae parasitizing marine molluscs from Patagonian coast, Argentina, based on literature sources and new records here presented. In total, cercariae of 31 species of marine digeneans, including 11 new records here presented, are known to infect 20 species of molluscs (14 in nine gastropods species; 17 in 11 bivalve species). These records include two species of Aporocotylidae, four Bucephalidae, one Fellodistomidae, five Gymnophallidae, one Hemiuroidea, four Lepocreadiidae, two Microphallidae, five Monorchiidae, one Notocotylidae, one Philophthalmidae, two Renicolidae, one Schistosomatidae and two Zoogonidae. For each digenean species, their hosts, habitat type, localities, infection site, prevalence, life cycle advances, and access numbers in helminthological collections and GenBank, when available, are detailed. Regarding the life cycles, eight were completely elucidated and four were partially elucidated. Moreover, there are molecular data for 15 species. The data here presented constitutes an advance in the parasites biodiversity knowledge and their life cycles.
\end{abstract}

Key words: parasites, bivalves, gastropods, diversity, cercariae, larvae, digenean

\section{INTRODUCTION}

The Patagonian Shelf extends for about 5,649 km along the Atlantic coast of South America from northern Uruguay $\left(33^{\circ} 51^{\prime} \mathrm{S}, 053^{\circ} 11^{\prime} \mathrm{W}\right)$ to the southern tip of Argentina, bordering with Chile $\left(54^{\circ} 55^{\prime} \mathrm{S}, 064^{\circ} 52^{\prime} \mathrm{W}\right)$. Its continental shelf is generally up to $100 \mathrm{~m}$ in depth, is the largest and one of the most productive ecosystems in the Southern Hemisphere (Miloslavich et al. 2011). In the Patagonian Shelf, two major marine currents coexist, the cold Malvinas and the warm Brazil currents. The Malvinas current originates in the Antarctic circumpolar current and carries a high nutrient load north along the Argentine coast. Biogeographically, the Patagonian Shelf is divided into two provinces, the Argentine and the Magellanic, that join around Peninsula Valdés. The
Argentine Biogeographic Province extends from $36^{\circ}$ to $43^{\circ} \mathrm{S}$ and the Magellanic Biogeographic Province extends from $43^{\circ}$ to $56^{\circ} \mathrm{S}$ (Miloslavich et al. 2011). The estimated mollusc species number is about 200 in the Argentine province and about 720 species in the Magellanic province (Bigatti and Penchaszadeh 2008). The great diversity of molluscs is given by the physiographic and climatic features of the Magellanic province, which is highly homogeneous and it has a lot of endemic taxa (Balech and Erlich 2008). Argentina's coast has mostly sandy beaches and some rocky formations. These rocky shores are dominated by two mussel species and by a diverse macroalgal community with an obvious tidal zonation.

Molluscs generally act as first intermediate hosts of digenean life cycles (Rohde 2005; Cremonte 2011) and their larval stages develop in the gonad and the digestive gland of them. The taxonomic identification of larval digenean is an important step towards the elucidation of their life cycles and the posterior use as a predictive tool in ecological studies (Nolan and Cribb 2005).

Lothar Szidat was largely responsible for inaugurating the study of marine helminthes in Argentina (Lunaschi et al. 2007). He recorded two bucephalid cercariae parasitizing the mussel Brachidontes rodrigezi (d'Orbigny) (Szidat 1963). Many freshwater cercariae (e.g., Ostrowski de Núñez 1974; Suriano and Martorelli 1983; Etchegoin et al. 1996; Martorelli and Etchegoin 1996; Martorelli et al., 2013) were recorded; however the knowledge about marine cercariae is scarce. Five isolated records were published by Ageitos de Castellanos (1961), Morris (1984), Martorelli (1991) and Szidat (1963, 1965). Since 2009 to date several advances in studies of cercariae from marine gastropods were done in Argentina by our research group. Despite the importance of digeneans in coastal marine areas, the data are scattered in literature and only 18 species of larval digenean in molluscs have been recorded (eight infecting six species of gastropods and 10 infecting six species of bivalves) in the Southwestern Atlantic coast. The aim of this study 
is to present information and new data of occurrence of larval digenean found in the commonest gastropods and bivalves inhabiting coast from Argentina.

\section{MATERIALS AND METHODS}

A survey of the scientific data on larval digeneans found in marine molluscs from the Argentine coast was done through scientific papers between 1961 and 2014. Furthermore, we included new records from our research group. Specimens described by our research group were sampled during low tide on several occasions along different sites from the coast of Patagonia, Argentina. Molluscs were transported to the laboratory and placed in small flasks filled with seawater at room temperature $\left(20-23^{\circ} \mathrm{C}\right)$. Emerged cercariae were studied alive, stained with neutral red or Nile blue under light microscope before being fixed. After $48 \mathrm{~h}$, all molluscs were necropsied in order to detect prepatent infections and to study larval stages. The morphology was studied alive and photographed. Several naturally emerged cercariae were killed with heated seawater, immediately fixed with 10\% formalin (Cribb and Bray 2010), stained with acetic carmine, dehydrated through ascendant ethanol series, cleared with methylsalicylate and mounted on glass slides with Canada balsam. Prevalences were calculated following Bush et al. (1997). Specimens of cercariae were deposited in the Parasitological Collection of Centro Nacional Patagónico (CONICET), Puerto Madryn, Chubut province, Argentina. A sample of cercariae specimens were preserved in ethanol $96 \%$ and frozen for molecular analyses. These analyses were performed on ribosomal DNA (mainly ITS1 e ITS2 regions) using standard techniques of DNA extraction, amplification by PCR and DNA sequencing (see protocols in Cremonte et al. 2013, 2015).

The taxonomy of digenean species is in accordance with Cable (1954, 1956, 1963), Holliman (1961), Yamaguti (1975) and Stunkard (1983). The taxonomic identity of the mollusc hosts were updated according to WoRMS (2015) and the species names were corroborated by malacological specialists. The marine cercaria species found in molluscs from Argentina are presented in alphabetic order for each family; each record contains information of the species name, authority and year, the name of mollusc host species, authority and family; the habitat where the respective life cycles of the digeneans occur (inter- or subtidal); the Argentine localities where of the molluscs were collected. Moreover, data related to prevalence of infection and life cycle were presented when it is available. If material deposited in helminthological collections or molecular sequences GenBank of other larval stages were available, the respective accession numbers were clarified between parentheses.
The acronyms for the Helminthological Collections are: Helminthological Collection of the Museo de La Plata (MLP), La Plata, Buenos Aires province, Argentina; National Parasitological Collection of the Museo Argentino de Ciencias Naturales "Bernardino Rivadavia" (MACN-Pa), Buenos Aires province, Argentina; Parasitological Collection of the Centro Nacional Patagónico (CNP-PAR), Puerto Madryn, Chubut province, Argentina.

The records were based on naturally infected molluscs. For most of the species listed in this study, cercariae emerge from the molluscs in aquatic environment; in other cases, when the life cycle is abbreviated, the information is provided in the section of the life cycle advances.

\section{RESULTS}

A total of 31 cercariae were identified infecting 20 species of molluscs, one at superfamily level, 14 at family level, 10 at genera level and six at species level. From 31 marine cercariae, 14 are infecting nine gastropods species and 17 are infecting 11 bivalve species. Figure 1 shows the localities where the cercariae were recorded.

\section{Phylum Platyhelminthes Gegenbaur, 1859 \\ Subphylum Neodermata (Ehlers, 1995) \\ Class Trematoda Rudolphi, 1808 \\ Subclass Digenea Carus, 1863}

\section{Aporocotylidae Odhner, 1912}

\section{Aporocotylidae gen. et sp. 1}

Host: Ensis macha (Molina) (Bivalvia: Pharidae).

HABITAT: Sandy subtidal.

CERCARIAN GROUP: Furcocercous.

Locality: La Tapera Beach, San José Gulf $\left(42.33^{\circ} \mathrm{S}\right.$, 064.55 ${ }^{\circ} \mathrm{W}$ ), Chubut province.

INFECTION SITES: Gonad, digestive gland and gills.

PREVALENCE: $1 / 30$ (3.3\%).

MATERIAL DEPOSITED: CNP-PAR 59 (histological section). REFERENCE: Vázquez et al. (2013).

\section{Aporocotylidae gen. et sp. 2}

Host: Amiantis purpurata (Molina) (Bivalvia:Veneridae). HABITAT: Sandy subtidal.

CERCARIAN gROUP: Furcocercous.

Locality: El Molino Beach, San Matías Gulf (40.82 ${ }^{\circ}$, $064.73^{\circ} \mathrm{W}$ ), Río Negro province.

INFECTION SITES: Gills and a kidney in high intensities of infection.

PREVAlence: $3 / 690$ (0.43\%).

MATERIAL DEPOSITED: MLP 6288, 6289; CNP- Par 31, 32.

REFERENCE: Gilardoni et al. (2011b). 


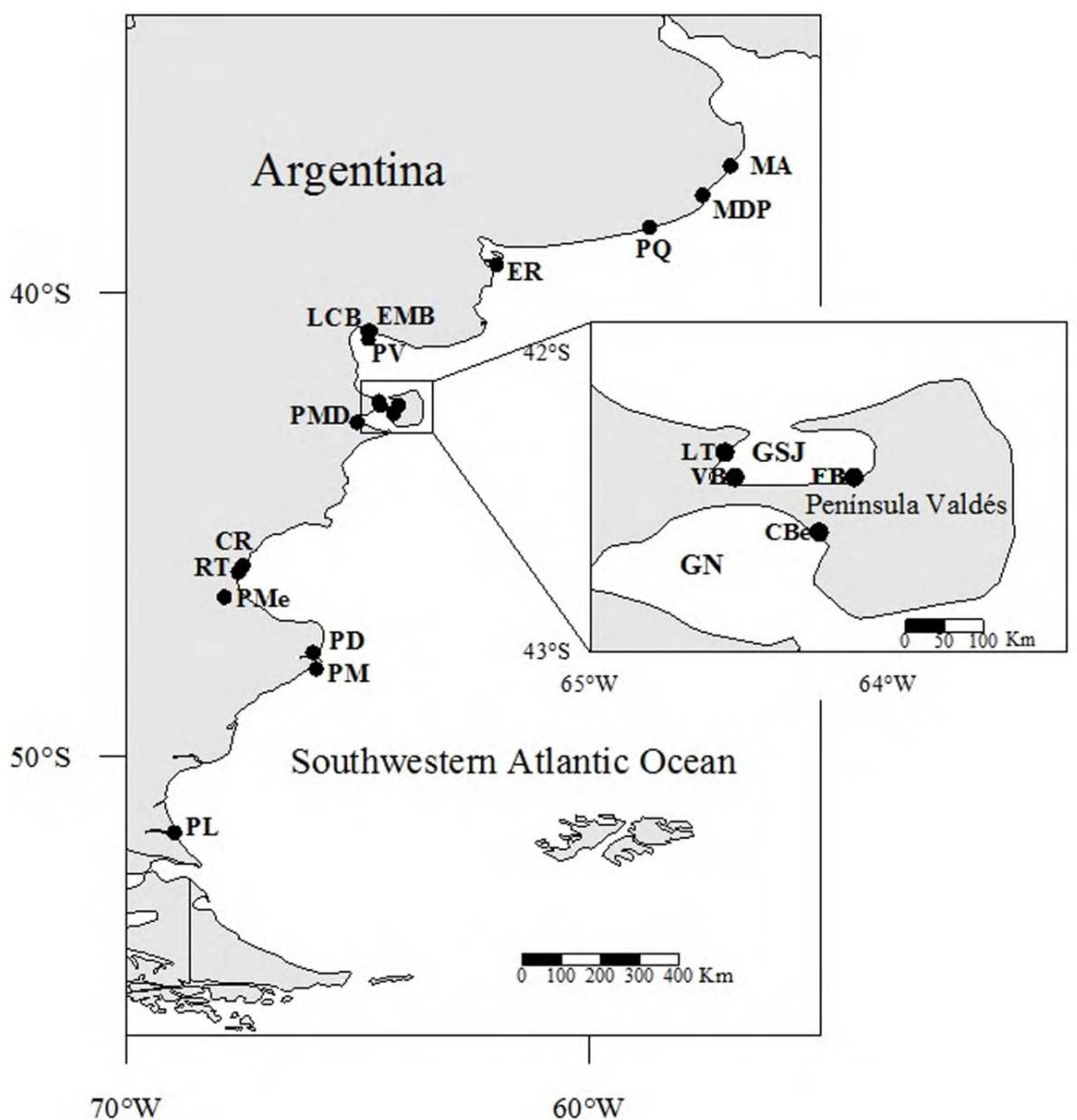

Figure 1. Map of localities where molluscs were found infected with marine larval digeneans in Argentina. Localities abbreviations: $\mathrm{CBe}$, Colombo Beach; CR, Comodoro Rivadavia; EMB, El Molino Beach; ER, El Rincón; FB, Fracasso Beach; LCB, La Conchilla Beach; LT, La Tapera; MA, Mar Azul; MDP, Mar del Plata; PD, Puerto Deseado; PMD, Puerto Madryn; PQ; Puerto Quequén; PL, Punta Loyola; PM, Punta Maqueda; PMe, Punta Medanosa; PV, Punta Villarino; RT, Rada Tilly; VB, Villarino Beach.

\section{Bucephalidae Poche, 1907}

\section{Bucephalidae gen. et sp. (New record)}

Hosts: Mytilus edulis (Linnaeus), Perumytilus purpuratus (Lamarck), Aulacomya atra (Molina) (Bivalvia: Mytilidae).

HABITAT: Rocky intertidal.

CERCARIan group: Gasterostome.

Localites: Puerto Madryn (PMD) $\left(42.78^{\circ} \mathrm{S}, 065.02^{\circ}\right.$ $\mathrm{W})$, Comodoro Rivadavia (CR) $\left(45.87^{\circ} \mathrm{S}, 067.48^{\circ} \mathrm{W}\right)$, Chubut province.

INFECTION SITES: Gonad and digestive gland.

PreValences: 1/15 (6.67\%) (M. edulis) (CR), 16/151 (10.6\%) (P. purpuratus) (CR, PMD), 1/15 (6.67\%) (A. atra) (CR).

MATERIAL DEPOSITED: CNP-PAR 73 (PMD).

\section{Bucephalus von Baer, 1827}

\section{Bucephalus sp. 1}

Host: Brachidontes rodriguezii (d'Orbigny) (Bivalvia: Mytilidae).
HABITAT: Rocky intertidal.

CERCARIAN GROUP: Gasterostome.

Localities: Puerto Quequén (PQ) $\left(38.58^{\circ} \mathrm{S}, 058.70^{\circ}\right.$ $\mathrm{W})$ and Mar del Plata (MDP) $\left(37.88^{\circ} \mathrm{S}, 057.55^{\circ} \mathrm{W}\right)$,

Buenos Aires province.

INFECTION SITES: Gonad, digestive gland and base gills. PREVALENCE: 17/1398 (1.2\%) (MDP). MATERIAL DEPOSITED: CNP-PAR 72 (MDP).

REFERENCES: Szidat (1963), Morris (1984).

\section{Bucephalus sp. 2}

Host: Mytilus edulis (Linnaeus) (Bivalvia: Mytilidae).

HABITAT: Rocky intertidal.

CERCARIAN GROUP: Gasterostome.

Localities: Mar del Plata (MDP) $\left(37.88^{\circ} \mathrm{S}, 057.55^{\circ} \mathrm{W}\right)$,

Puerto Quequén (PQ) $\left(38.58^{\circ} \mathrm{S}, 058.70^{\circ} \mathrm{W}\right)$ and

El Rincón (ER) $\left(39.73^{\circ} \mathrm{S}, 60.83^{\circ} \mathrm{W}\right)$, Buenos Aires province.

INFECTION SITES: Gonad and digestive gland.

PREVAlences: 4/650 (o.62\%) (MDP), 39/1,089 (3.58\%) (PQ), 5/262 (1.91\%) (ER).

MATERIAL DEPOSITED: MACN-Pa 144 (1-7) (MDP); 
CNP-PAR 68 (ER).

References: Ageitos de Castellanos (1961), Szidat (1965), Morris (1984).

\section{Prosorhynchus Odhner, 1905}

\section{Prosorhynchus sp.}

Host: Brachidontes rodriguezii (d'Orbigny) (Bivalvia: Mytilidae).

HABITAT: Rocky intertidal.

CERCARIAN GROUP: Gasterostome.

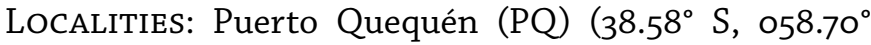
$\mathrm{W})$ and Mar del Plata (MDP) $\left(37.88^{\circ} \mathrm{S}, 057.55^{\circ} \mathrm{W}\right)$, Buenos Aires province.

INFECTION SITES: Gonad, digestive gland and base gills. PREVALENCE: 28/1000 (2.8\%) (MDP).

MATERIAL DEPOSITED: MACN-Pa 145 (PQ); CNP-PAR 71 (MDP).

REFERENCES: Szidat (1963).

\section{Fellodistomidae Nicoll, 1909 \\ Monascus Looss, 1907}

\section{Monascus filiformis (Rudolphi, 1819)}

Host: Ennucula puelcha (d'Orbigny) (Bivalvia: Nuculidae). HABITAT: Sandy subtidal.

CERCARIAN GROUP: Furcocercous.

LocAlity: Mar del Plata (37.88 S, $\left.057.55^{\circ} \mathrm{W}\right)$, Buenos Aires province.

INFECTION SITES: Gonad and digestive gland.

PREVALENCE: $1 / 23$ (4.35\%).

LIFE CYCLE: Hydromedusae Clytia sp. (Campanulariidae), Liriope tethraphyla Chamisso \& Eysenhardt (Geryoniidae), Eucheilota ventricularis McCrady (Lovenellidae), Aglauropsis kawari Moreira \& Yamashita (Olindiidae) and chaetognathe Sagitta sp. (Sagittidae) as second intermediate hosts; marine teleost fish Trachurus lathami Nichols (Carangidae) as definitive host.

MATERIAL DEPOSITED: CNP-PAR 67.

REFERENCES: Girola et al. (1992), Martorelli and Cremonte (1998).

\section{Gymnophallidae Odhner, 1905}

\section{Gymnophallidae gen. et sp. 1 (New record)}

Host: Lasaea adansoni (Gmelin) (Bivalvia: Lasaeidae).

HABITAT: Among byssi of mytilids or among Balanus sp.

(Balanidae) cirripedians, rocky intertidal.

CERCARIAN group: Furcocercous.

LocAlities: Comodoro Rivadavia (CR) (45.87 $\mathrm{S}, 067.48^{\circ}$

W), Chubut province and Puerto Deseado (PD) $\left(47.75^{\circ}\right.$

$\mathrm{S}$, $\left.065.97^{\circ} \mathrm{W}\right)$, Santa Cruz province.

INFECTION SITES: Gonad, also in mantle, gills and nephridia in high intensities of infection.

PreVAlences: 4/33 (12.5\%) (CR), 6/517 (1.16\%) (PD).
Material Deposited: CNP- Par 45 (PD).

\section{Gymnophallidae gen. et sp. 2 (New record)}

Host: Neolepton cobbi (Cooper \& Preston) (Bivalvia:

Neoleptonidae).

HABITAT: Sandy intertidal.

CERCARIAN GROUP: Furcocercous.

LocAlity: Puerto Deseado (47.75 ${ }^{\circ}$ S, 065.97 W), Santa

Cruz province.

INFECTION SITE: Gonad.

PREVAlENCE: $1 / 142$ (0.7\%).

MATERIAL DEPOSITED: CNP- Par 46.

\section{Gymnophallidae gen. et sp. 3 (New record)}

Host: Gaimardia trapesina (Lamarck) (Bivalvia: Gaimardiidae).

HABITAT: On fronds of the giant kelp, Macrocystis pyrifera (Linnaeus) (Laminariaceae), shallow subtidal.

CERCARIAN Group: Furcocercous.

LocAlity: Puerto Deseado ( $\left.47.75^{\circ} \mathrm{S}, 065.97^{\circ} \mathrm{W}\right)$, Santa

Cruz province.

INFECTION SITES: Gonad and digestive gland.

PREVALENCE: 8/504 (1.59\%).

MATERIAL DEPOSITED: CNP- Par 47.

\section{Bartolius Cremonte, 2001}

\section{Bartolius pierrei Cremonte, 2001}

Host: Darina solenoides (King) (Bivalvia: Mactridae).

HABITAT: Sandy intertidal.

CERCARIAN GROUP: Furcocercous.

LocAlity: Fracasso Beach, San José Gulf $\left(42.42^{\circ} \mathrm{S}\right.$, $\left.064.12^{\circ} \mathrm{W}\right)$, Chubut province.

INFECTION SITES: Gonad, digestive gland and kidney.

PREVALENCE: 3/6o (5\%).

LIFE CYCLE: Same clam D. solenoides as second intermediate host (metacercaria encyst in the extrapallial cavity); kelp gull Larus dominicanus Lichtenstein (Laridae) and Calidris canutus rufa (Linnaeus) (Scolopacidae) as definitive hosts.

MATERIAL DEPOSITED: MLP 5020, 5064-5069; CNP-PAR 58 (cercaria), 1 (metacercaria), 12 (adult).

References: Cremonte (2001), Cremonte (2004).

\section{Gymnophalloides Fujita, 1925}

\section{Gymnophalloides nacellae Cremonte, Pina,} Gilardoni, Rodrigues, Chai \& Ituarte, 2013

Host: Gaimardia trapesina (Lamarck) (Bivalvia: Gaimardiidae).

HABITAT: On fronds of the giant kelp Macrocystis pyrifera

(Linnaeus) (Laminariaceae), shallow subtidal.

Cercarian group: Furcocercous.

LocAlity: Puerto Deseado (47.75 S, 065.97 W), Santa

Cruz province.

INFECTION SITE: Gonad and digestive gland. 
PREVALENCE: $10 / 504$ (1.98\%).

LIFE CYCLE ADVANCES: The gastropod Nacella (Patinigera) magellanica (Nacellidae) as second intermediate host.

MATERIAL DEPOSITED: CNP-PAR 48 (cercaria), 50, 51 (metacercaria).

GenBANK NUMBER: KF575168 (sporocyst), JN381025 (metacercaria) (ITS1).

REFERENCES: Ituarte et al. (2005), Cremonte et al. (2013).

\section{Hemiuroidea Looss, 1899}

\section{Hemiuroidea fam., gen. et sp.}

Host: Siphonaria lessonii Blainville (Gastropoda: Siphonariidae).

HABITAT: Rocky intertidal.

Cercarian group: Cystophorous.

Localities: Fracasso Beach (FB) $\left(42.42^{\circ} \mathrm{S}\right.$, $064.12^{\circ}$ W), Puerto Madryn (PMD) $\left(42.78^{\circ} \mathrm{S}, 065.02^{\circ} \mathrm{W}\right)$, Comodoro Rivadavia (CR) $\left(45.87^{\circ} \mathrm{S}, 067.48^{\circ} \mathrm{W}\right)$, Chubut province and Puerto Deseado (PD) $\left(47.75^{\circ} \mathrm{S}\right.$, $\left.065.97^{\circ} \mathrm{W}\right)$, Santa Cruz province.

INFECTION SITES: Gonad, digestive gland, glandular complex associated with the mantle, connective tissue of mantle and secondary gill in high intensities of infection.

PREVAlENCES: 11/223 (5\%) (PF), 5/1,050 (o.48\%) (PDM), 6/500 (1.2\%) - 50/500 (10\%) (CR), 9/186 (4.84\%) (PD).

MATERIAL DEPOSITED: MLP 5738 (CR), CNP- Par 26 (PD).

GENBANK NUMBER: KF451931 (sporocyst) (PMD) (ITS1).

REFERENCES: Alda and Martorelli (2009), Gilardoni et al. (2011a).

\section{Lepocreadiidae Odhner, 1905}

\section{Lepocreadiidae gen. et sp. 1 (New record)}

Host: Crepipatella dilatata (Lamarck) (Gastropoda: Calyptraeidae).

HABITAT: Rocky intertidal.

CERCARIAN Group: Ophthalmo trichocercous.

Locality: Puerto Deseado $\left(47.75^{\circ} \mathrm{S}\right.$, $\left.065.97^{\circ} \mathrm{W}\right)$, Santa Cruz province.

INFECTION SITES: Gonad and also digestive gland in high intensities of infection.

PREVAlenCE: $10 / 266$ (3.76\%).

MATERIAL DEPOSITED: CNP-PAR 52.

GenBANK NUMBER: KF451932, KF451933 (redia) (PD) (18S, ITS1, ITS2, 28S).

\section{Lepocreadiidae gen. et sp. 2 (New record)}

Host: Pareuthria plumbea Philippi (Gastropoda: Buccinidae).

HABITAT: Rocky intertidal.

Cercarian group: Ophthalmo trichocercous.

Locality: Puerto Deseado $\left(47.75^{\circ} \mathrm{S}, 065.97^{\circ} \mathrm{W}\right)$, Santa Cruz province.
INFECTION SITES: Gonad and also digestive gland in high intensities of infection.

PREVAlenCE: $16 / 458$ (3.49\%).

MATERIAL DEPOSITED: CNP-PAR 53.

GeNBANK NUMBER: KF451934, KF451935 (redia) (18S, ITS1, ITS2, 28S).

\section{Opechona Looss, 1907}

\section{Opechona sp. 1}

Host: Buccinanops monilifer (Kiener) (Gastropoda: Nassariidae).

HABitat: Sandy subtidal.

Cercarian group: Ophthalmo trichocercous.

LocAlitY: Mar del Plata $\left(37.88^{\circ} \mathrm{S}\right.$, $\left.057.55^{\circ} \mathrm{W}\right)$, Buenos Aires province.

INFECTION SITES: Gonad and digestive gland.

LIFE CYCLE ADVANCES: Jellyfish, Olindias sambaquiensis Müller (Olindiidae) as second intermediate host.

REFERENCE: Martorelli (1991).

\section{Opechona sp. 2}

Host: Buccinanops cochlidium (Dillwyn) (Gastropoda: Nassariidae).

HABITAT: Sandy subtidal.

CERCARIAN GROUP: Ophthalmo trichocercous.

Locality: Villarino Beach, San José Gulf $\left(42.42^{\circ} \mathrm{S}\right.$, 064.31 $\left.1^{\circ} \mathrm{W}\right)$, Chubut province.

INFECTION SITES: Gonad and also digestive gland in high intensities of infection.

PREVALENCE: $66 / 426$ (15.5\%).

MATERIAL DEPOSITED: CNP-PAR 39.

GENBANK NUMBER: KF451938, KF451939 (redia) (18S, ITS1, ITS2, 28S).

REFERENCE: Averbuj and Cremonte (2010).

\section{Microphallidae Travassos, 1920 Maritrema (Maritrema) Nicoll, 1907}

\section{Maritrema sp. 1}

Host: Crepipatella dilatata (Lamarck) (Gastropoda: Calyptraeidae).

HABITAT: Rocky intertidal.

Cercarian group: Monostome xiphidiocercaria.

Localities: Puerto Madryn (PMD) $\left(42.78^{\circ} \mathrm{S}, 065.02^{\circ}\right.$ W), Chubut province and Puerto Deseado (PD) ( $\left.47.75^{\circ} \mathrm{S}, 065.97^{\circ} \mathrm{W}\right)$, Santa Cruz province.

INFECTION SITES: Gonad and digestive gland, also foot and mantle in high intensities of infection.

PreValences: 557/1,665 (33.45\%) (PMD), 44/266 (16.5\%) (PD). MATERIAL DEPOSITED: MLP 6285 (PMD), CNP-PAR 28 (PMD).

GENBANK NUMBER: KCO12521 (sporocyst) (PMD) (ITS1, $5.8 \mathrm{~S}, \mathrm{ITS} 2$ ).

REFERENCE: Gilardoni et al. (2011a). 
Maritrema madrynense Diaz \& Cremonte, 2010

Hosts: Siphonaria lessonii Blainville and S. lateralis Gould (Gastropoda: Siphonariidae).

HABITAT: Rocky intertidal.

CERCARIAn group: Monostome xiphidiocercaria.

LocAlities: Fracasso Beach (FB) $\left(42.42^{\circ} \mathrm{S}, 064.12^{\circ} \mathrm{W}\right)$, Puerto Madryn (PMD) $\left(42.78^{\circ} \mathrm{S}, 065.02^{\circ} \mathrm{W}\right)$, Comodoro Rivadavia (CR) ( $45.87^{\circ} \mathrm{S}$, $\left.067.48^{\circ} \mathrm{W}\right)$, Rada Tilly (RT) $\left(46.55^{\circ} \mathrm{S}, 067.88^{\circ} \mathrm{W}\right)$, Chubut province; Punta Maqueda (PM) $\left(46.02^{\circ} \mathrm{S}, 067.58^{\circ} \mathrm{W}\right)$ and Puerto Deseado (PD) $\left(47.75^{\circ} \mathrm{S}, 065.97^{\circ} \mathrm{W}\right)$, Santa Cruz province.

INFECTION SITES: Gonad and digestive gland, glandular pallial complex and gills in high intensities of infection.

PreVAlENCES: 39/394 (10\%) (FB), 94/1,050 (8.95\%) (PMD), 34/500 (6.73\%)-405/500 (81\%) (CR, RT, PM), $17 / 423(4.02 \%)(\mathrm{PD})$.

LIFE CYCLE: Same gastropod S. lessonii (metacercaria encyst inside sporocyst); crab Cyrtograpsus altimanus Rathbun (Crustacea) and isopod Idothea baltica (Pallas) (Crustacea) as second intermediate hosts; kelp gull Larus dominicanus Lichtenstein (Laridae) as definitive host.

MATERIAL DEPOSITED: MLP 5736, 6284 (CR, PM, RT); CNP-PAR 27 (cercaria), 74 (metacercaria) (PMD), 11 (adult) (FB).

GenBANK NUMBER: KC222O22 (sporocyst), KC222023, $\mathrm{KC} 222024$ (metacercaria), $\mathrm{KF}_{575167}$ (adult) (PMD) (ITS1, 5.8S, ITS2).

REFERENCES: Alda and Martorelli (2009); Diaz and Cremonte (2010); Gilardoni et al. (2011a).

\section{Monorchiidae Odhner, 1911}

\section{Monorchiidae gen. et sp. 1}

Host: Amiantis purpurata (Molina) (Gastropoda: Veneridae).

HABITAT: Sandy intertidal and subtidal.

CERCARIAN GROUP: Microcercous.

Localities: Mar Azul (MA) $\left(37.25^{\circ} \mathrm{S}, 056.95^{\circ} \mathrm{W}\right)$, Buenos Aires province and Punta Villarino (PV) $\left(40.83^{\circ} \mathrm{S}\right.$, $064.75^{\circ}$ W) San Matías Gulf, Río Negro province.

INFECTION SITES: Gonad and digestive gland, also foot, mantle, gills and around intestine in high intensities of infection.

PreVAlenCes: $16 / 17$ (94.1\%) (MA), 19/220 (8.8\%) (PV).

MATERIAL DEPOSITED: MLP 4808, 4809 (cercariae and metacercariae) (PV), 4810 (sporocysts) (MA); CNPPAR 69 (MA).

REFERENCE: Cremonte et al. (2001).

\section{Monorchiidae gen. et sp. 2}

Host:Amiantis purpurata (Molina) (Bivalvia:Veneridae). HABITAT: Sandy intertidal.

CERCARIAN Group: Ophthalmo-leptocerca.
LOCALITY: La Conchilla Beach ( $40.82^{\circ} \mathrm{S}$, $\left.064.78^{\circ} \mathrm{W}\right)$, San Matías Gulf, Río Negro province.

INFECTION SITES: Gonad and digestive gland, also foot and mantle in high intensities of infection.

PREVAlenCE: $1 / 690$ (0.14\%).

MAterial Deposited: MLP 6286, 6287; CNP- Par 29, 30. REFERENCE: Gilardoni et al. (2011b).

\section{Postmonorcheides Szidat, 1950}

\section{Postmonorcheides maclovini Szidat, 1950 (New record)}

Host: Lasaea adansoni (Gmelin) (Bivalvia: Lasaeidae).

HABITAT: Among byssi of mytilids or among cirripedians Balanus sp. (Balanidae), rocky intertidal.

CERCARIAN GROUP: Leptocercous.

Locality: Puerto Deseado $\left(47.75^{\circ} \mathrm{S}, 065.97^{\circ} \mathrm{W}\right)$, Santa Cruz province.

INFECTION SITE: Gonad.

PREVALENCE: $18 / 648$ (2.78\%).

LIFE CYCLE: Same clam L. adansoni act as second intermediate host (metacercaria encyst inside sporocyst); mullet Eleginops maclovinus (Cuvier) (Eleginopidae) as definitive host.

MATERIAL DEPOSITED: CNP- Par 61 (cercaria), 62 (adult). GENBANK NUMBER: KC920685 (sporocyst with cercariae and metacercariae), KC920684 (adult) (ITS1).

REFERENCE: Szidat (1950) (adult).

\section{Proctotrema Odhner, 1911}

\section{Proctotrema bartolii Carballo, Laurenti \& Cremonte,} 2011

Host: Darina solenoides (King) (Bivalvia: Mactridae).

HABITAT: Sandy intertidal.

Cercarian group: Microcercous.

Localities: Fracasso Beach (FB) $\left(42.42^{\circ} \mathrm{S}, 064.12^{\circ} \mathrm{W}\right)$, Colombo Beach ( $\mathrm{CBe})\left(42.60^{\circ} \mathrm{S}\right.$, 064.23 $\left.\mathrm{W}\right)$, Chubut province; Punta Medanosa (PMe) $\left(48.10^{\circ} \mathrm{S}, 065.90^{\circ}\right.$ W), Punta Loyola (PL) $\left(51.63^{\circ} \mathrm{S}, 068.97^{\circ} \mathrm{W}\right)$, Santa Cruz province.

INFECTION SITES: Gonad, digestive gland, also gills in high intensities of infection.

PREVAlENCES: $60 / 80$ (75\%)- 80/80 (100\%) (FB), 15/15 (100\%) (CBe), 15/15 (100\%) (PMe), 15/15 (100\%) (PL).

LIFE CYCLE ADVANCES: Same clam D. solenoides as second intermediate host (metacercariae encyst mainly in incurrent siphon); silversides Odonthestes smitti (Lahille) and O. nigricans (Richardson) (Pisces: Atherinopsidae) and mullet Eleginops maclovinus (Cuvier) (Eleginopidae) as definitive hosts.

MATERIAL DEPOSITED: CNP-PAR 2, 3, 4, 5 (FB).

REFERENCE: Gilardoni et al. (2013).

\section{Proctotrema sp. (New record)}

Host: Gaimardia trapesina (Lamarck) (Bivalvia: Gaimardiidae). 
HABITAT: On fronds of the giant kelp Macrocystis pyrifera (Linnaeus) (Laminariaceae), shallow subtidal.

Cercarian group: Microcercous.

LocAlitY: Puerto Deseado $\left(47.75^{\circ} \mathrm{S}, 065.97^{\circ} \mathrm{W}\right)$, Santa Cruz province.

INFECTION SITES: Gonad and digestive gland.

PREVALENCE: 8/692 (1.15\%).

LIFE CYCLE ADVANCES: Same clam G. trapesina as second intermediate host (metacercaria encyst in foot); nototheniids Patagonotothen cornucola (Richardson) and P. sima (Richardson) (Nototheniidae) as definitive hosts.

MATERIAL DEPOSITED: CNP-PAR 42 (cercaria), 43 (metacercaria), 44 (adult).

GENBANK NUMBER: KP765717 (sporocyst), KP765716 (adult) (ITS1).

\section{Notocotylidae Lühe, 1909}

\section{Notocotylidae gen. et sp. (New record)}

Host: Nacella (Patinigera) magellanica (Gmelin) (Gastropoda: Nacellidae).

HABITAT: Rocky intertidal.

CERCARIAN GROUP: Monostomate.

LocAlity: Puerto Deseado $\left(47.75^{\circ} \mathrm{S}, 065.97^{\circ} \mathrm{W}\right)$, Santa Cruz province.

INFECTION SITES: Gonad and digestive gland.

PREVALENCE: $1 / 306$ (0.33\%).

MATERIAL DEPOSITED: CNP-PAR 57.

GENBANK NUMBER: KF656705 (metacercaria) (ITS1).

\section{Philophtalmidae Looss, 1899 \\ Parorchis Nicoll, 1907}

\section{Parorchis sp.}

Host: Trophon geversianus (Pallas) (Gastropoda: Muricidae).

HABITAT: Rocky intertidal.

CERCARIAN GROUP: Echinostome.

LocAlities: Fracasso Beach (FB) $\left(42.42^{\circ} \mathrm{S}, 065.12^{\circ} \mathrm{W}\right)$, Puerto Madryn (PMD) $\left(42.78^{\circ} \mathrm{S}, 065.02^{\circ} \mathrm{W}\right)$, Chubut province and Puerto Deseado (PD) $\left(47.75^{\circ} \mathrm{S}, 065.97^{\circ}\right.$ W), Santa Cruz province.

INFECTION SITES: Gonad and digestive gland.

PrevalenCES: $17 / 427$ (4\%) (FB), 6/689 (o.87\%) (PMD), $1 / 151$ (o.66\%) (PD).

LIFE CYCLE ADVANCES: Second intermediate host absent (cercaria encyst in the substrate); kelp gull Larus dominicanus and white-rumped sandpiper Calidris fuscicollis (Vieillot) (Scolopacidae) as definitive hosts.

MATERIAL DEPOSITED: MLP 6282 (PMD), CNP-PAR 24, 25 (cercaria) (PMD), 15 (adult) (FB).

GenBANK NUMBER: KF451927 (sporocyst) (PMD), KF451928, KF451929, KF451930 (adult) (PD) (ITS1, 5.8S, ITS2, 28S).

REFERENCES: Diaz et al. (2011); Gilardoni et al. (2011a).

\section{Renicolidae Dollfus, 1939}

\section{Renicolidae gen. et sp. 1}

Host: Trophon geversianus (Pallas) (Gastropoda: Muricidae).

HABITAT: Rocky intertidal.

CERCARIAN GROUP: Xiphidiocercaria.

Localities: Fracasso Beach (FB) $\left(42.42^{\circ} \mathrm{S}, 064.12^{\circ} \mathrm{W}\right)$, Puerto Madryn (PMD) $\left(42.78^{\circ} \mathrm{S}, 065.02^{\circ} \mathrm{W}\right)$, Chubut province and Puerto Deseado (PD) $\left(47.75^{\circ} \mathrm{S}, 065.97^{\circ}\right.$ W), Santa Cruz province.

INFECTION SITES: Gonad and digestive gland.

Prevalences: 2/427 (0.5\%) (FB), 2/689 (0.29\%) (PMD), 7/151 (4.64\%) (PD).

LIFE CYCLE ADVANCES: Mussel Mytilus edulis as second intermediate host; kelp gull Larus dominicanus Lichtenstein (Laridae) as definitive host.

MATERIAL DEPOSITED: MLP 6278-6279 (PMD), CNP-PAR 20 (PMD).

GenBANK NUMBeR: KF358774 (sporocyst) (PMD), KF425522 (metacercaria) (CR) (ITS1).

REFERENCES: Gilardoni et al. (2011a).

\section{Renicolidae gen. et sp. 2 (New record)}

Host: Nacella (Patinigera) magellanica (Gmelin) (Gastropoda: Nacellidae).

HABITAT: Rocky intertidal.

CERCARIAN GROUP: Xiphidiocercaria.

LocAlity: Puerto Deseado $\left(47.75^{\circ} \mathrm{S}\right.$, $\left.065.97^{\circ} \mathrm{W}\right)$, Santa Cruz province.

INFECTION SITE: Digestive gland.

PREVALENCE: $3 / 306$ (0.98\%).

MATERIAL DEPOSITED: CNP-PAR 55.

GenBANK NUMBER: KF358775 (sporocyst) (ITS1).

\section{Schistosomatidae Stiles \& Hassall, 1898}

\section{Schistosomatidae gen. et sp.}

Host: Siphonaria lessonii Blainville (Gastropoda: Siphonariidae).

HABITAT: Rocky intertidal.

CERCARIAN GROUP: Distome furcocercous apharyngeate. LocalitiEs: Fracasso Beach (FB) $\left(42.42^{\circ} \mathrm{S}\right.$, $\left.064.12^{\circ} \mathrm{W}\right)$, Comodoro Rivadavia (CR) $\left(45.87^{\circ} \mathrm{S}, 067.48^{\circ} \mathrm{W}\right)$, Chubut province and Puerto Deseado (PD) $\left(47.75^{\circ} \mathrm{S}\right.$, $\left.065.97^{\circ} \mathrm{W}\right)$, Santa Cruz province.

INFECTION SITES: Gonad and digestive gland, mucous gland and in the vicinity of seminal vesicle.

Prevalences: 5/394 (1.3\%) (PF), 6/500 (1.2\%), 31/500 (6.1\%) (CR), 1/186 (0.54\%) (PD).

MATERIAL DEPOSited: MLP 5737 (CR), CNP-PAR 56 (FB).

REFERENCES: Alda and Martorelli (2009).

\section{Zoogonidae Odhner, 1902}

Diphterostomum Stossich, 1903 
Diphterostomum brusinae (Stossich, 1889)

Host: Buccinanops globulosus (Kiener) (Gastropoda: Nassariidae).

HABITAT: Sandy intertidal and subtidal.

CERCARIAN group: Tailess xiphidiocercaria.

LocalitiEs: Fracasso Beach (FB) $\left(42.42^{\circ} \mathrm{S}, 064.12^{\circ} \mathrm{W}\right)$, Puerto Madryn (PMD) $\left(42.78^{\circ} \mathrm{S}, 065.02^{\circ} \mathrm{W}\right)$, Chubut province.

INFECTION SITES: Gonad and also digestive gland in high intensities of infection.

Prevalences: 9/430 (2.1\%) (Intertidal sample) (FB), 3/59 (5.1\%) (Subtidal sample) (PMD).

LIFE CYCLE ADVANCES: Same gastropod B. globulosus (metacercaria encyst inside sporocyst) and polychaete Kinbergonuphis dorsalis (Ehlers) (Onuphidae) as second intermediate host; reef fish Pinguipes brasilianus Cuvier (Pinguipedidae) as definitive host.

MATERIAL DEPOSITED: MLP 6280, 6281 (PMD); CNPPAR 21, 23 (cercaria), 22 (metacercaria) (PMD).

GenBANK NUMBER: KF358772 (sporocyst), KF483875 (adult) (PMD) (ITS1, 5.8S, ITS2).

REFERENCES: Timi et al. (2008), Gilardoni et al. (2011a).

\section{Zoogonus Looss, 1901}

\section{Zoogonus sp. (New record)}

Host: Pareuthria plumbea Philippi (Gastropoda: Buccinidae).

HABITAT: Rocky intertidal.

CERCARIAN GROUP: Tailess xiphidiocercaria.

Locality: Puerto Deseado $\left(47.75^{\circ} \mathrm{S}, 065.97^{\circ} \mathrm{W}\right)$, Santa Cruz province.

INFECTION SITES: Gonad and digestive gland.

PREVALENCE: $1 / 458$ (0.22\%).

MATERIAL DEPOSITED: CNP-PAR 54.

GENBANK NUMBER: KF358773 (sporocyst) (ITS1, 5.8S, ITS2).

\section{DISCUSSION}

This bibliographic survey, with new record, presents the diversity of marine digenean cercariae in molluscs from Argentine coast over 54 years of parasitological studies. A total of 31 cercariae were known to parasitize 20 species of molluscs; 14 of which parasitized nine gastropods species and 17 were parasitized 11 bivalve species.

However, the number of cercariae identified at generic (10) or specific level (6) is relatively small in comparison with a great number of adults digenean known (226) from vertebrates hosts, 125 from fish (e.g., Kohn et al. 2007; Braicovich and Timi 2008; Timi et al. 2008; Timi and Lanfranchi 2009), 95 from birds (e.g., Lunaschi et al. 2007; Diaz and Cremonte 2010) and six from marine mammals (e.g., Lunaschi et al. 2007). However, from Argentina, there are few species of molluscs recorded (20) with digenean larvae, despite that the diversity of Argentine marine molluscs is high (849 species) (Miloslavich et al. 2011).

This study is the third checklist of marine larval trematodes from South America. The two previous checklists recorded marine and freshwater cercariae from Chile (Muñoz and Olmos 2008) and Brazil (Pinto and Melo 2013), and these includes records since 1980 and 1912, respectively. The Chilean checklist summarized 63 species of digeneans; nine of them are larval trematodes (sporocysts or rediae with cercariae) parasitizing seven molluscan species and five of them are from marine molluscs. About 500 digeneans are known in vertebrates from Brazil. From 46 larval trematodes species recorded in 25 molluscan species in Brazil, only five are marine. The records of larval trematodes from freshwater molluscs (at least 95 species) from Argentina are much higher (e.g., Ostrowski de Núñez 1992; Ostrowski de Núñez et al. 1997; Flores and Semenas 2008; Martorelli et al. 2013; Alda and Martorelli 2014), than Chile and Brazil. However, the number of records from marine molluscs is higher than Chile and Brazil ( 31 digenean species from 20 molluscs). We found 12 families, corresponding to Gymnophallidae, Monorchiidae, Bucephalidae, Lepocreadiidae, Aporocotylidae, Microphallidae, Renicolidae, Zoogonidae, Fellodistomidae, Hemiuridae, Philophtalmidae and Schistosomatidae. From Chilean marine cercariae, two species belong to Fellodistomidae, one each to Bucephalidae, Gymnophallidae and Plagiorchiidae. From Brazilian marine cercariae, three species belong to Bucephalidae, one to Cyathocotylidae and one (Cercaria maritima) to an unidentified family.

Studies of larval digeneans are necessary to elucidate life cycles and to link cercariae to their respective adult parasites. Moreover, morphological descriptions should be complemented with molecular data, because the majority of larvae can only be identified to family by their morphologically. On the other hand, the distribution of records is not uniform throughout the country mainly because most studies were made in a few Patagonian localities. We hope that this review can stimulate new studies on marine larval digeneans and contribute to the biodiversity knowledge from South American marine parasites.

\section{ACKNOWLEDGEMENTS}

The authors thank Dr. Gregorio Bigatti from CENPAT, Puerto Madryn, Argentina, who helped us with literature on mollusc diversity of the Argentine coast; Dr. Diego Zelaya from University of Buenos Aires, Argentina, for checking all mollusc species; Dr. Lía Lunaschi from MLP, La Plata, Argentina and Dr. Fabián Tricárico from MACN, Buenos Aires, Argentina, for providing data on deposited material; the Erasmus Mundus Foundation for the Eurotango Fellowship to CG, and to Dr. Pedro 
Rodrigues and Dr. Susana Pina from ICBAS, Porto, Portugal, for their helping in molecular analyses. The present study was funded by the CONICET (PIP 20132015 to Drs. Julia Diaz \& Florencia Cremonte) and the ANPCyT (PICT 2013-1702). Authors are members of CONICET.

\section{LITERATURE CITED}

Ageitos de Castellanos, Z. 1961. Estados larvales de TrematodaBucephalidae en el mejillón Mytilus platensis. Notas del Museo de La Plata 20 (188): 21-24.

Alda, P. and S.R. Martorelli. 2009. Larval Digeneans of the Siphonariid Pulmonates Siphonaria lessoni and Kerguelenella lateralis and the Flabelliferan Isopod Exosphaeroma sp. from the Intertidal Zone of the Argentinean Sea. Comparative Parasitology 76 (2): 267-272. doi: $10.1654 / 4381.1$

Alda, P. and S.R. Martorelli. 2014. Larval trematodes infecting the South-American intertidal mud snail Heleobia australis (Rissooidea : Cochliopidae). Acta Parasitologica 59 (1): 50-67. doi: 10.2478/s11686-014-0209-3

Averbuj, A. and F. Cremonte. 2010. Parasitic castration of Buccinanops cochlidium (Gastropoda: Nassariidae) caused by a lepocreadiid digenean in San José Gulf, Argentina. Journal of Helminthology 84 (4): 381-389. doi: 10.1017/Soo22149X10000052

Balech, E. and M.D. Ehrlich. 2008. Esquema biogeográfico del Mar Argentino. Revista de Investigación y Desarrollo Pesquero 19: 45-75.

Bigatti, G. and P.E. Penchaszadeh. 2008. Invertebrados del mar Patagónico, diagnóstico de la problemática actual y potencial de su conservación y manejo. Estado de Conservación del Mar Patagónico 105-133.

Braicovich, P.E. and J.T. Timi. 2008. Parasites as biological tags for stock discrimination of the Brazilian flathead Percophis brasiliensis in the south-west Atlantic. Journal of Fish Biology 73 (3): 557-571. doi: 10.1111/j.1095-8649.2008.01948.x

Bush, A.O., K.D. Lafferty, J.M. Lotz and A.W. Shostak. 1997. Parasitology meets ecology on its own terms: Margolis et al. revisited. Journal of Parasitology 83 (4): 575-583. http://www. jstor.org/stable/3284227

Cable, R.M. 1954. A new marine cercaria from the Woods Hole region and its bearing on the interpretation of larval types in the Fellodistomatidae (Trematoda: Digenea). Biological Bulletin 106 (1): 15-20. http://www.biolbull.org/content/106/1/15

Cable, R.M. 1956. Marine cercariae of Puerto Rico. Scientific Survey of Puerto Rico and the Virgin Islands 16: 491-577.

Cable, R.M. 1963. Marine cercariae from Curaçao and Jamaica. Zeitschrift für Parasitenkunde 23: 429-469.

Cremonte, F. 2001. Bartolius pierrei n. g., n. sp. (Digenea: Gymnophallidae) from the Península Valdés, Argentina. Systematic Parasitology 49 (2): 139-147. doi: 10.1023/A:1010668604300

Cremonte, F. 2004. Life cycle and geographic distribution of the gymnophallid Bartolius pierrei (Digenea) on the Patagonian coast, Argentina. Journal of Natural History 38 (13): 1591-1604. doi: 10.1080/0022293031000156187

Cremonte, F. 2011. Enfermedades de moluscos bivalvos de interés comercial causadas por metazoos; pp. 333-396, in: A. Villalba and A. Figueras (eds). Enfermedades de moluscos bivalvos de interés en Acuicultura. Madrid: Fundación Observatorio Español de Acuicultura.

Cremonte, F., M.A. Kroeck and S.R. Martorelli. 2001. A new monorchiid (Digenea) cercaria parasitising the purple clam Amiantis purpurata (Veneridae) from the Southwest Atlantic Ocean, with notes on its gonadal effect. Folia Parasitologica 48 (3): 217-223. doi: 10.14411/fp.2001.035
Cremonte, F., S. Pina, C. Gilardoni, P. Rodrigues, J. Chai and C. Ituarte. 2013. A new species of gymnophallid (Digenea) and an amended diagnosis of the genus Gymnophalloides Fujita, 1925 Journal of Parasitology 99 (1): 85-92. doi: 10.1645/GE-2909.1

Cremonte, F., C. Gilardoni, S. Pina, P. Rodrigues and C. Ituarte. 2015. Revision of the family Gymnophallidae Odhner, 1905 (Digenea) based on morphological and molecular data. Parasitology International 64 (2): 202-10. doi: 10.1016/j.parint.2014.12.003

Cribb, T.H. and R.A. Bray. 2010. Gut wash, body soak, blender and heat -fixation: approaches to the effective collection, fixation and preservation of digenean of fishes. Systematic Parasitology 76 (1): 1-7. doi: 10.1007/s11230-010-9229-Z

Diaz, J.I. and F. Cremonte. 2010. Development from metacercaria to adult of a new species of Maritema (Digenea: Microphallidae) parasitic in the kelp gull, Larus dominicanus, from the Patagonian coast, Argentina. Journal of Parasitology 96 (4): 740-745. doi: 10.1645/GE-2343.1

Diaz, J.I., F. Cremonte and G.T. Navone. 2011. Helminths of the kelp gull, Larus dominicanus, from the northern Patagonian coast. Parasitology Research 109 (6): 1555-1562. doi: 10.1007/soo436011-2396-2

Etchegoin, J.A., S.R. Martorelli and N.H. Sardella. 1996. Nuevo registro de Microphallus szidati Martorelli, 1986 (Digenea: Microphallidae) en Mar Chiquita (Buenos Aires, Argentina). Neotrópica 42 (107-108): 117-118.

Flores, V.R. and L. Semenas. 2008. Larval Digenean community parasitizing the freshwater snail, Chilina dombeyana (Pulmonata: Chilinidae) in Patagonia, Argentina, with special reference to the notocotylid Catatropis chilinae. Journal Parasitology 94 (2): 305-313.

Gilardoni, C., M.C. Carballo and F. Cremonte. 2013. The life cycle and geographical distribution of the monorchiid Proctotrema bartolii (Digenea) in the clam Darina solenoides from the Patagonian coast, Argentina. Journal of Helminthology 87 (4): 392-399. doi: 10.1017/So022149X12000569

Gilardoni, C., J.A. Etchegoin, J.I. Diaz and F. Cremonte. 2011. a) A survey of larval digeneans in the commonest intertidal snails from Northern Patagonian coast, Argentina. Acta Parasitologica 56 (2): 163-179. doi: 10.2478/s11686-011-0021-2

Gilardoni, C., G. Posadas, M.A. Kroeck and F. Cremonte. 2011. b) Monorchiid and aporocotylid cercariae (Digenea) parasitising the purple clam Amiantis purpurata (Bivalvia, Veneridae) from the Southwestern Atlantic coast. Acta Parasitologica 56 (4): 385-391. doi: 10.2478/s11686-011-0074-2

Girola, C.V., S.R. Martorelli and N.H. Sardella. 1992. Presencia de metacercarias de Monascus filiformis (Digenea, Fellodistomidae) en hidromedusas del Océano Atlántico Sur. Revista Chilena de Historia Natural 65: 409-415.

Holliman, R.B. 1961. Larval digenean from Apalachee Bay area, Florida, with a checklist of known marine cercariae arranged in a key to their superfamilies. Tulane Studies in Zoology 9 (1): 1-74.

Ituarte, C., F. Cremonte and D.G. Zelaya. 2005. Parasite-mediated shell alterations in Recent and Holocene sub-Antarctic bivalves: the parasite as modeler of host reaction. Invertebrate Biology 124 (3): 220-229. doi: 10.1111/j.1744-7410.2005.00021.x

Kohn, A., B.M.M. Fernández and S.C. Cohen. 2007. South American trematode parasites of fishes. Rio de Janeiro: Ministério da Saúde, FIOCRUZ, Fundação Oswaldo Cruz. 318 pp.

Lunaschi, L.I., F. Cremonte and F.B. Drago. 2007. Checklist of digenean parasites of birds from Argentina. Zootaxa 1403: 1-36.

Martorelli, S.R. 1991. Primera cita de una cercaria tricocerca parásita de Dorsanum moniliferum (Mollusca: Buccinidae) para el Atlántico Sud-Occidental. Aportes al conocimiento de su ciclo de vida. Neotrópica 37 (97): 57-65.

Martorelli, S.R. and F. Cremonte. 1998. A proposed three-host life-history of Monascus filiformis (Rudolphi, 1819) (Digenea: 
Fellodistomidae) in the Southwest Atlantic. Canadian Journal of Zoology 76 (6): 1198-1203. doi: 10.1139/z98-039

Martorelli, S.R. and J.A. Etchegoin. 1996. Cercarias de la superfamilia Opistorchioidea en Heleobia conexa (Mollusca: Hydrobiidae) de la albufera Mar Chiquita. Neotrópica 42 (107-108): 61-67.

Martorelli, S.R., P. Alda, P. Marcotegui, L.F. La Sala and M.M. Montes. 2013. Larval digeneans in Biomphalaria snails from the Salto Grande Dam area in the Uruguay River. Publicación del Laboratorio de Helmintos y Parásitos de Crustáceos del CEPAVE 1-13.

Miloslavich, P., E. Klein, J.M. Díaz, C. E. Hernández, G. Bigatti, L. Campos, F. Artigas, J. Castillo, P. E. Penchaszadeh, P. E. Neill, A. Carranza, M. V. Retana, J. M. Díaz de Astarloa, M. Lewis, P. Yorio, M. L. Piriz, D. Rodríguez, Y. Yoneshigue-Valentin, L. Gamboa and A. Martín. 2011. Marine biodiversity in the Atlantic and Pacific coasts of South America: knowledge and gaps. PLOS ONE 6 (1): 1-43. doi: 10.1371/journal.pone.0014631

Morris, M.R. 1984. Estados larvales de digenean digeneos en moluscos marinos Mytilus platensis d'Orb. y Barchydontes rodriguezii d'Orb. Revista del Museo de La Plata (Nueva Serie) 13(135): 65-73.

Muñoz, G. and V. Olmos. 2008. Revisión bibliográfica de especies endoparásitas y hospedadoras de sistemas acuáticos de Chile. Revista de Biología Marina y Oceanografía 43(2): 173-245.

Nolan, M.J. and T.H. Cribb. 2005. The use and implications of ribosomal DNA sequencing for the discrimination of digenean species. Advances in Parasitology 60: 101-163. doi: 10.1016/ Soo65-308X(05)60002-4

Ostrowski de Núñez, M. 1974. Fauna de agua dulce de la República Argentina. II. Cercaria perteneciente a la superfamilia Opistorchioidea y parte de su ciclo evolutivo (Trematoda). Physis, Sección B 33 (86): 1-9.

Ostrowski de Núñez, M. 1992. Trematoda. Familias Strigeidae, Diplostomidae, Clinostomidae, Schistosomatidae, Spirochiidae y Bucephalidae; pp. 1-55, in: Ageitos de Castellanos, Z., S. Coscarón and S. Miquel (eds). Fauna de agua dulce de la República Argentina. Vol. 9, Fasc. 1 Furcocercarias. La Plata: PROFADU (CONICET).

Ostrowski de Núñez, M., M.I. Hamann and A. Rumi. 1997. Estudios de trematodes larvales en Biomphalaria spp. (Mollusca, Planorbidae) de la localidad de San Roque, Provincia de Corrientes, Argentina. Physis Sección B 54(126-127): 7-15.

Pinto H.A. and A.L. Melo. 2013. A checklist of cercariae (Trematoda: Digenea) in molluscs from Brazil. Zootaxa 3666(4): 449-475. doi: 10.11646/zootaxa.3666.4.3

Rohde, K. 2005. Marine Parasitology. Wallingford: University of New England: CSIRO Plublishing. $565 \mathrm{pp}$.

Stunkard, H.W. 1983. The marine cercariae of the Woods Hole,
Massachusetts Region, a review and a revision. Biological Bulletin 164 (2): 143-162. doi: 10.2307/1541135

Suriano, D.M. \& S.R. Martorelli. 1983. Estudios parasitológicos en la albufera de Mar Chiquita, provincia de Buenos Aires, República Argentina. Neotrópica 29 (82): 195-207.

Szidat, L. 1950. Los parásitos del róbalo ("Eleginops maclovinus" Cuv. \& Val.). Primer Congreso Nacional de Pesquerías Marítimas e Industrias Derivadas 2: 235-270.

Szidat, L. 1963. Los parásitos de los mitílidos y los daños por ellos causados I. Los parásitos de los "mejillines", Brachydontes rodriguezii y Semimytilus algosus. Neotrópica 29 (9): 80-86.

Szidat, L. 1965. Los parásitos de los mitílidos y los daños por ellos causados II. Los parásitos de Mytilus edulis platensis (Orb.) (mejillón del plata). Comunicaciones del Museo Argentino de Ciencias Naturales "Bernardino Rivadavia" 1(1): 1-16.

Timi, J.T. and A.L. Lanfranchi. 2009. The metazoan parasite communities of the Argentinean sandperch Pseudopercis semifasciata (Pisces: Perciformes) and their use to elucidate the stock structure of the host. Parasitology 136 (10): 1209-1219. doi: 10.1017/So031182009990503

Timi, J.T., A.L. Lanfranchi, J.A. Etchegoin and F. Cremonte. 2008. Parasites of the Brazilian sandperch Pinguipes brasilianus Cuvier: a tool for stock discrimination in the Argentine Sea. Journal of Fish Biology 72(6): 1332-1342. doi: 10.1111/j.10958649.2008.01800.x

Vázquez, N., E.P. Bruno, F. Márquez, S. Van Der Molen, C. Gilardoni and F. Cremonte, 2013. A histopathological survey of the razor clam Ensis macha (Pharidae) along the Patagonian Argentina coast. Journal of Invertebrate Pathology 112: 253-259. doi: 10.1016/j.jip.2012.12.004

WoRMS (World Register of Marine Species). [2015]. Accessed at http://www.marinespecies.org, 27 January 2015.

Yamaguti, S. 1975. A synoptical review of life histories of digenetic digeneans of vertebrates with special reference to the morphology of their larval forms. Tokyo: Keigaku Publishing Co. $156 \mathrm{pp}$.

Authors' contribution statement: EB collected the parasites and wrote the manuscript; CG collected the parasites and oversaw the draft manuscript; GDG collected the parasites; FC identified the species and oversaw the draft manuscript.

Received: 3 March 2015

Accepted: 11 June 2015

Academic editor: Simone Chinicz Cohen 